\title{
NEW CASES OF RECONSTRUCTIBILITY OF SBT GRAPHS
}

\author{
Martin Dowd \\ 1613 Wintergreen Pl. \\ Costa Mesa, CA 92626, USA
}

\begin{abstract}
In earlier papers the author showed that all graphs which are not single-block trunk (SBT) graphs are reconstructible, and that two families of SBT graphs are reconstructible. Here some further families of SBT graphs are shown to be reconstructible.
\end{abstract}

AMS Subject Classification: 05C60

Key Words: reconstructibility, SBT graph

\section{Introduction}

Let $G$ be a graph. A limb in $G$ is a maximal induced subgraph, which is a tree. If $G$ is not a tree each $\operatorname{limb} L$ has a root $r$, which attaches it to the rest of the graph. Removing the subgraphs $L-r$ for the limbs $L$, the remaining induced subgraph is called the trunk.

If $G$ is not a tree, and the trunk is inseparable, $G$ will be called a singleblock trunk (SBT) graph. In [2] it is shown that if $G$ is not an SBT graph then $G$ is reconstructible. Let $n_{v}(G)\left(n_{e}(G)\right)$ denote the number of vertices (edges) of $G$. If $G$ is an SBT graph let $n_{p}(G)=n_{e}(G)-n_{p}(G)$; letting $B$ denote the block comprising the trunk of $G$, this equals $n_{e}(B)-n_{v}(B)$. In [3] it is shown that an SBT graph is reconstructible if $n_{p} \leq 1$. 
In this paper some results will be proved which imply that an SBT graph is reconstructible if $n_{p} \leq 2$. There are errors in [3], and new proofs will be given for $n_{p}=0$ and $n_{p}=1$ as well.

A graph $G$ with a vertex $v$ deleted is denoted $G_{v}$.

It has long been known ([1]) that if a graph $G$ has limbs, then the multiset of limbs with the attachment point marked, and the trunk, are reconstructible. By the size of a limb will be meant its number of edges, or the number of vertices other than the root. A limb of size 1 will be called a 1-limb.

The proof of lemma 11 of [2] is essentially a proof that the limbs are determined; it has an error, which will be corrected here. Namely, the claim is made that if in $G_{v}$ for all degree 1 vertices $v$ the limbs are $n$-limbs for some $n \geq 1$, then in $G$ the limbs are $n+1$-limbs. This is true if $n \geq 2$; suppose $n=1$. If there is a vertex $w$ of degree 2 such that in $G_{w}$ there are two components, one of which is an isolated vertex and the other of which has no degree 1 vertex, then the limbs of $G$ are a single path of length 2. If there is a vertex $w$ such that $G_{w}$ has 2 components which are isolated vertices, then then the limbs of $G$ are a single complete binary tree of height 1 . If neither of these hold, the limbs are 2 1-limbs.

Let $L_{d s}$ be the multiset of limbs of size $s$ attached to vertices of degree $d$ in the trunk.

Theorem 1. The multiset of $L_{d s}$ is reconstructible.

Proof. Except for the case of a single 1-limb, this is theorem 2 of [3]. In the case of a single 1-limb, $G_{v}$ where $v$ is the root of the 1-limb may be determined, and the degree of $v$ is determined.

Theorem 2. Let $G$ be an SBT graph. For given d, unless the set of sizes of the limbs attached to degree $d$ vertices of $B$ is an interval $[0, m]$ for some $m$, $G$ is reconstructible.

Proof. If there is a limb of size $s$ but none of size $s-1$ let $v$ be a leaf of a tree of size $s$. In $G_{v}$ there is a single limb of size $s-1$, and this may be replaced by the limb missing from $L_{d s}$. 


\section{Automorphisms}

Suppose $G$ is an SBT graph and $\alpha$ is an automorphism of $B$. Let $G^{\alpha}$ be the result of $\alpha$ 's action on $G$. That is, for a vertex $r$ of $B$, let $T_{r}$ (resp. $T_{r}^{\alpha}$ ) denote the tree rooted at $r$ in $G\left(\operatorname{resp} . G^{\alpha}\right)$; then $T_{r}^{\alpha}=T_{\alpha(r)}$.

Suppose $v$ is a degree 1 vertex of a limb of $G$. $G$ "lies over" $G_{v}$, meaning that there is a vertex $r_{0}$ (namely the root of the limb containing $v$ ), such that $T_{r v}$ equals $T_{r}$, except if $r=r_{0}$ it has $v$ deleted. $G$ lies over $G_{v}$ in some other way iff $G^{\alpha}$ lies over $G_{v}$, where $\alpha$ is a nontrivial automorphism of $B$. Say that $\alpha$ is allowable (for $v$ ) in this case.

Clearly, an automorphism $\alpha$ of $G$ is allowable. Although they will not be used, some additional facts will be stated. Suppose $G^{\alpha}$ lies over $G_{v}$, where $r_{1}$ is the vertex of $B$ such that $T_{r_{1}}^{\alpha}$ does not equal $T_{r_{1}, v}$. It is possible that $r_{1}=r_{0}$.

Suppose $r_{0}, \alpha\left(r_{0}\right), \ldots, \alpha^{l-1}\left(r_{0}\right)$ is the orbit of $r_{0}$. For $0<i<l$, as long as $\alpha^{i}\left(r_{0}\right) \neq r_{1}, T_{\alpha^{i}\left(r_{0}\right)}=T_{\alpha^{i-1}\left(r_{0}\right)}$. This is so for all such $i$ iff $r_{1}=r_{0}$. In any case, $r_{1}$ is in the orbit of $r_{0}$. For $r$ in some other orbit, $T_{\alpha(r)}=T_{r} . \alpha$ is an automorphism of $G$ iff $r_{1}=r_{0}$. Otherwise, $T_{\alpha^{j}\left(r_{0}\right)}=T_{r_{0}, v}$ for $i \leq j<l$ where $\alpha^{i}\left(r_{0}\right)=r_{1}$.

Theorem 3. Suppose $G$ is an SBT graph. If $B$ is rigid and there are at least 2 degree 1 vertices then $G$ is reconstructible.

Proof. Let $v$ range over the degree 1 vertices. The $G_{v}$ may be "aligned" along $B$. $G$ is then the union of the $G_{v}$.

\section{L-paths}

Recalling some definitions of [4], an inseparable graph $A$ is said to be a subdivision of an inseparable graph $B$ if $A$ is obtained from $B$ by dividing edges by adding degree 2 vertices. Note that $n_{p}(A)=n_{p}(B)$. $B$ is said to be S-minimal if it is not a subdivision of another graph. It is shown in [4] that an inseparable graph $B$ is a subdivision of a unique S-minimal graph $B_{r}$.

If $G$ is a graph let $n_{v}^{d}(G)$ denote the number of vertices of degree $d$, and $n_{\bar{v}}^{\geq d}(G)$ the number of degree at least $d$. For an inseparable graph $B n_{\bar{v}}^{\geq 3}(B)=$ $n_{\bar{v}}^{\geq 3}\left(B_{r}\right)$.

$n_{\bar{v}}^{\geq 3}(B)=0$ iff $B$ is a cycle iff $n_{p}(B)=0$ iff $B_{r}$ is a 3 -cycle. If $n_{\bar{v}}^{\geq 3}(B) \neq 0$ then $n_{\bar{v}}^{\geq 3}(B) \geq 2$. In this case, by a T-path in $B$ is meant a maximal path all 
of whose interior vertices have degree 2 in $B$. A T-path in an SBT graph $G$ is a T-path in its trunk $B$. An L-path in $G$ is a T-path, with the limbs attached to its interior vertices adjoined, and its end points marked. The length of an L-path $P$ is the length of its T-path, and the size of $P$ is the sum of the sizes of the limbs, or the number of vertices not on the T-path.

An L-path $P$ of length $l$ may be oriented by labeling the vertices of its T-path from 0 to $l$, from one end to the other. If $P$ is an oriented L-path let $P^{r}$ denote $P$ with the opposite orientation, and for a degree 1 vertex $v$ of a limb let $P_{v}$ have the inherited orientation. Let $r_{0}<\cdots<r_{t}$ be the roots of the limbs, let $T_{r}$ denote the limb rooted at vertex $r$, and let $T_{r_{i}}$ be the limb containing $v$. Let $\rho$ denote reversal of $P$. Say that $\rho$ is allowable if $P^{\rho}$ lies over $P_{v}$. If $\rho$ is allowable say that it is type 1 if $T_{r_{i}}$ equals $T_{l-r_{i}}$, and type 2 if $T_{r_{i}, v}$ equals $T_{l-r_{i}}$.

Theorem 4. Suppose $P$ is an L-path of size $s \geq 2$ and $v$ is a degree 1 vertex of a limb. Suppose $\rho$ is allowable. If $\rho$ is type 1 then $P^{\rho}=P$, otherwise $\rho$ is not allowable for $w$ where $w$ is the end of a degree 1 vertex of $T_{l-r_{i}}$.

Proof. Straightforward.

\section{4. $B$ a cycle}

Suppose $G$ is an SBT graph and its trunk $B$ is a cycle.

Lemma 5. If $G$ has limbs of size $\geq 2$ then $G$ is reconstructible.

Proof. Let $u$ be a degree 1 vertex of a 1 -limb $T$, and let $v$ be a degree 1 vertex of a limb of size $\geq 2$. Let $R$ be $B$ with the roots marked; this may be determined from $G_{v}$. Let $R_{u}$ be $B$ with the roots marked, other than that of the limb $T$ containing $u$; this may determined from $G_{u}$.

If there is a rotation which is an automorphism of $R$ let $\alpha$ be the one of highest order. $G$ may be reconstructed from $G_{v}$, by locating the orbit with $T$, and then the location of $T$, and adding a 1-limb.

If $R$ is rigid than $R$ fits over $R_{u}$ in only one way, whence the location in $G_{v}$ of $T$ may be determined and $G$ reconstructed.

In the remaining case the automorphism group of $R$ is generated by a single reflection, whose axis may be determined in any $G_{v}$. $G$ may be divided into 
two subgraphs $P_{1}$ and $P_{2}$, each containing the vertices on one side of the axis, where a vertex on the axis and its attached limb if any are in both $P_{i}$. Let $r$ denote the number of limbs of size $\geq 2$. If $r \geq 3$ then $P_{1}$ and $P_{2}$ in $G$ may be determined by considering the $G_{v}$.

Indeed, let $r_{1} \leq r_{2}$ be the number of limbs on each $P_{i}$. By considering the $G_{v}$ these may be determined, and also the side $P_{2}$ with $r_{2}$ limbs. The other side $P_{1}$ is also easily determined, unless $r_{2}=r_{1}+1$. In this case, let $Q$ be the degree 1 vertices of limbs of size $\geq 2$ along $P_{2}$. Among the $P_{i}$ (two each) of the $G_{v}$ where $v \in Q$, only one subgraph occurs $|Q|$ times, and this is $P_{1}$.

In the remaining cases, $r \leq 2$; the reflection symmetry of $R$ will not be used.

Suppose $r=2$. Let $T_{1}, T_{2}$ be the two limbs of size $\geq 2$. By considering the $G_{u}$ the distance from $T_{1}$ to $T_{2}$, and the number of 1-limbs in between, may be determined. If all 1-limbs are between, $G$ may be reconstructed from $G_{v}$ where $v$ is a degree 1 vertex of $T_{i}$ where the size of $T_{i}$ is as small as possible.

Otherwise, the L-path $P_{0}$ between $T_{1}$ and $T_{2}$ may be determined from the $G_{u}$. Let $S$ be the 1-limb roots not on $P_{0}$. $P_{0}$ may be located in $G_{u}$ for any $u$ in a 1-limb with root in $S$. For $i=1,2$ let $P_{i}$ be the paths from the root of $T_{i}$, away from $P_{0}$, containing the 1-limb roots closest to $T_{i}$. For $i=1,2,3$ let $p_{i}$ be the number of 1-limbs along $P_{i}$. Say that $u$ is on $P_{i}$ if its 1-limb is.

If $p_{0} \geq 2 G$ may be reconstructed from the $G_{u}$ where $u$ is on $P_{0}$, by theorem 4 .

Suppose $p_{0} \leq 1$. Suppose $p_{1}+p_{2} \geq 3$. By considering the $G_{u}$ it may be determined if all of $S$ lies along a single $P_{i}$. If this is the case $G$ may be reconstructed from the $G_{u}$ for $u$ in a 1-limb with root in $S$, by taking the union. Otherwise, $P_{0} \cup P_{1}$ and $P_{0} \cup P_{2}$ may be determined, and $G$ reconstructed from these. This is clear except for the case $p_{2}=p_{1}+1$. In this case, among the paths (two each) of the $G_{u}$ where $u$ is on $P_{2}$, only one path occurs $p_{2}$ times, and this is $P_{1}$.

Suppose $1 \leq p_{1}+p_{2} \leq 2$. If $p_{0}=1$ then $P_{1}$ and $P_{2}$ may be determined. There are 4 cases $p_{0}, p_{1}+p_{2}$ as follows.

1,2. Whether the 1-limbs other than the one on $P_{0}$ are on the same $P_{i}$ may be determined. $G$ may then be reconstructed as when $p_{1}+p_{2} \geq 3$.

1,1. Let $d_{0}$ be the distance from $T_{1}$ to $T_{2}$. In $G_{v}$ where $v$ is in the $T_{i}$ of least size, there is exactly 1 limb at distance $d$ from the $T_{i}$ of greatest size, with a 1-limb in between. $G$ is now readily reconstructed.

0,2 . Let $d_{0}$ be the distance from $T_{1}$ to $T_{2}, d_{1}, d_{2}$ the distance from the 1-limbs to the nearest $T_{i}$, and $b$ the cycle length. The possibilities $d_{0}, d_{1}, d_{2}-d_{1}, b-$ $d_{2}-d_{0}$ and $d_{0}, d_{1}, b-d_{2}-d_{1}-d_{0}, d_{2}$ may be compared to the cyclic distances 
determined from $R$. Trying all 8 possible orderings of the second possibility, there is an ambiguity in only 3 cases, and in all of them $d_{2}=\left(b-d_{0}\right) / 2$. Whether this occurs is readily determined, and $G$ reconstructed in this case as well.

0,1 . Let $d_{0}$ be the distance from $T_{1}$ to $T_{2}$. Suppose $v$ is in a $T_{i}$ of least size. If there is a single 1-limb at distance $d_{0}$ from $T_{3-i}$ in $T_{v}$ then $G$ may be reconstructed from $G_{v}$. Suppose there are 2 such. If the distance between them is $d_{0} G$ may be reconstructed from $G_{u}$. Otherwise $G$ may be reconstructed from $G_{v}$ where $v$ is in $T_{3-i}$.

Suppose $r=1$. Defined $P_{1}, P_{2}, p_{1}, p_{2}$ as in the case $r=2$. If $p_{1}+p_{2} \geq 3 G$ may be reconstructed as in the case $r=2$.

If $p_{1}+p_{2}=2$ proceed as in the 0,2 subcase of the case $r=2$. The possibilities are $d_{1}, d_{2}-d_{1}, b-d_{2}$ and $d_{1}, b-d_{2}-d_{1}, d_{2}$. Trying all 6 possible orderings of the second possibility, there is an ambiguity in only 2 cases, and in both of them $d_{2}=b / 2$. Whether this occurs is readily determined, and $G$ reconstructed in this case as well.

If $p_{1}+p_{2}=1 G$ may be reconstructed from $G_{v}$.

Lemma 6. Suppose the limbs of $G$ are 1 or more 1-limbs; then $G$ is reconstructible.

Proof. Let $b$ be the length of the cycle. Let $v$ range over the vertices of $B$. If for any $G_{v}$ the maximum path length is $b$ or $b-2, G$ may be reconstructed from such a $G_{v}$. Otherwise, $b=4 t$ where $t>0$, and numbering $B$ circularly from 0 , there are 1 -limbs attached to vertices numbered $i$ where $i \equiv 0,1 \mathrm{mod}$ 4.

It is a question of interest whether, when there are at least 31 -limbs, $G$ can be reconstructed from the $G_{u}$. The author has verified this for 3 or 4 1-limbs, and conjectures that it is true in general.

Theorem 7 . If $B$ is a cycle then $G$ is reconstructible.

Proof. If there are no limbs the claim is readily verified. Otherwise it follows by lemmas 5 and 6 . 


$$
\text { 5. } n_{\bar{v}}^{\geq 3}(B)=2
$$

From hereon, for an SBT graph $G$ with trunk $B$, say that a vertex of $B$ of degree $\geq 3$ is an E-vertex. Let $n_{v}^{E}$ denote the number of them, i.e., $n_{v}^{\geq 3}(B)$. Call a limb attached to an E-vertex an E-limb. Call the remaining limbs Ilimbs. A vertex $v$ will be said to be determining if $G_{v}$ can be determined, and $G$ reconstructed from $G_{v}$.

If $n_{v}^{E}(B)=2$ then $n_{p}(B) \geq 1$ and $B$ consists of two vertices joined by $n_{p}(B)+2$ T-paths, at most one of which is an edge; and $B_{r}$ consists of two vertices joined by an edge and $n_{p}(B)+1 \mathrm{~T}$-paths of length 2 . Let $d_{E}=n_{p}(B)+2$ denote the E-vertex degree. A vertex $v$ is an E-vertex iff $G_{v}$ is acyclic.

Theorem 8. If $n_{v}^{E}=2$ then $G$ is reconstructible.

Proof. Let $s_{1} \leq s_{2}$ be the sizes of the E-limbs. If $\left\{s_{1}, s_{2}\right\}$ is not $\{0\}$ or $\{0,1\}$ the claim follows by theorem 2. If the L-paths all have size 0 then an E-vertex with an E-limb if any attached is determining. Suppose neither of these cases holds. Say that an augmented L-path is an L-path, with the E-limb if any attached to its root. Let $u$ range over degree 1 vertices.

The L-path sizes may be determined. If $v$ is the degree 1 vertex of an E-limb they may be determined from $G_{v}$; suppose there are no E-limbs, and let $n$ be the number of degree 1 vertices. If $n \geq 3$ the size multiset may be determined from those for the $G_{u}$, by a standard method (see e.g. the proof of lemma 11 of [2]). If $n=1$, or if there is a single limb of size 2, the claim is trivial. If there are 21 -limbs let $v$ be the root of a limb; the size list is $\left\{0^{d_{E}-2}, 1,1\right\}$ if $G_{v}$ has a cycle with a limb, else $\left\{0^{d_{e}-1}, 2\right\}$.

Let $m$ be the minimum nonzero size, and let $r$ be the number of L-paths of size $m$. There is more than 1 limb iff either $r>1$, or $r=1$ and there are L-paths of size $>m$. It will next be shown that in this case, the multiset of augmented L-paths is determined.

Indeed, it is determined if a vertex $v$ is a non-root vertex of a limb of an L-path of size $m$; let $Q$ denote the set of these. Choose a $v \in Q$; the augmented L-paths of size $>m$ are those for $G_{v}$. If $r>1$, consider the size $m$ augmented L-paths for the $G_{u}$, where $u$ ranges over $Q$. Each size $m$ augmented L-path appears $(r-1) m$ times.

If $r=1$ and there are L-paths of size greater than $m$, choose a vertex $v$ in the L-path of size $m$. Let $u$ range over the non-root limb vertices of the Lpaths of size greater than $m$. For each such $u$, let $A_{u}$ be the multiset of size $m$ augmented L-paths in $G_{u}$. For each such $u$ we can also determined the multiset 
$B_{u}$ of size $m$ augmented L-paths in $G_{v u}$. The size $m$ augmented L-path $C$ of $G$ may be determined by taking the multiset union of the $A_{u}$ and of the $B_{u}$; the former will equal the latter, with some number of additional elements which are all copies of $C$.

Let $v$ be a degree 1 vertex of an L-path of size $m$. If $m>1$ the size 0 augmented L-paths are those of $G_{v}$. Otherwise the augmented L-path $C$ of size 1 missing from $G$ is determined, and the length 0 augmented L-paths of $G$ are those of $G_{v}$, with $C_{v}$ deleted.

If there is an E-limb, $G$ is determined by its multiset of augmented L-paths; suppose not.

Suppose there is an $s \geq 2$ such that there is an L-path $P$ of size $s$ but none of size $s-1$. Then it is determined whether $v$ is a degree 1 vertex of a limb of $P$, and if so $P_{v}$ may be found in $G_{v}$. $G$ may be reconstructed using theorem 4.

Suppose $d_{E} \geq 4$. Since there are I-limbs there must be an L-path of size 1; the root of its 1-limb is determining.

Suppose there is a single L-path $P$ of size $m \geq 2$, and there is an E-vertex. Then $P$ may be found in $G_{v}$ where $v$ is the degree 1 vertex of the E-limb. $G$ may be reconstructed using theorem 4 .

If $P$ has a single limb of size $\geq 2$ a degree 1 vertex of the limb is determining.

Suppose there is a single L-path $P$ of size $m \geq 3$, and there is no E-limb. Let $P_{0}$ be the sub-path of $P$ containing the limbs, with limbs at the ends. The length of $P_{0}$ may be determined from the $G_{v}$ where $v$ ranges over the degree 1 vertices. $P_{0}$ may then be determined from $G_{w}$ where $w$ is a degree 2 vertex on a size 0 L-path of length $\geq 2$. $G$ may be reconstructed using theorem 4 applied to $P_{0}$.

The remaining cases are as follows, where ijk are the L-path sizes, and E indicates an E-limb also.

002, 2 1-limbs. Let $v$ be a vertex such that $G_{v}$ is a cycle with a single limb attached. Since the lengths of the T-paths are known the length of the T-path $P$ is determined from the length of the cycles; and also the location on the cycle of the other E-vertex. Since it is known whether $v$ has a 1-limb attached, $G$ may be reconstructed from $G_{v}$.

001. Let $v$ be a vertex such that $G_{v}$ is a cycle with a single limb attached, or no limbs. The argument is similar to the preceding case; if there is no 1-limb the E-vertices may be spaced on the cycle.

001E. Let $v$ be a vertex such that $G_{v}$ is a cycle with a single limb attached. The argument is similar to the preceding case.

In the remaining cases, $d_{E}=3$ and the L-paths are determined. If an Lpath of length 2 has a limb let $s$ be the smallest size of such; an end vertex of 
a size $s$ limb on an L-path of length 2 is determining. Thus it may be assumed that the L-paths have length $\geq 3$.

A vertex $u$ will be specified, as that where $G_{u}$ has a cycle with certain limbs. One of these will be recognizable as having an E-vertex $x$ as its root. Let $P_{1}$ be the path containing $u$, and $l_{1}$ its length; $l_{1}$ equals the sum of the lengths of the T-paths, minus the length the cycle. Knowing $l_{1}$ and the size of $P_{1}, P_{1}$ may be reconstructed by connecting an end of a new edge up to a uniquely determined vertex of the limb rooted at $x$, and possibly also an isolated vertex. Knowing $P_{1}$ the other two paths $P_{2}$ and $P_{3}$ are known, and the other E-vertex $y$ can be located on the cycle. $G$ may be reconstructed by attaching the other end of the new edge of $P_{1}$ to the cycle at $y$. Let $l_{2}, l_{3}$ be the lengths of $P_{2}, P_{3} ; y$ may be either at distance $l_{2}$ from $x$ counterclockwise around the cycle, or at distance $l_{3}$.

011. Let $u$ be such that $G_{u}$ has 1 -limb and a limb of size $\geq 2, x$ being the root of the latter. Let $P_{2}$ be the size 0 path, let $d, d^{\prime}$ be the distances from the root of the 1-limb of $P_{3}$ to the ends, and let $e$ be the distance from $x$ on the cycle in $G_{u}$, to the 1-limb on the cycle. There are two possibilities for the location of the other E-vertex in $G_{u}$; for both possibilities for $y$ to be allowable, $l_{2}<e$ must hold, and both $e$ and $e-l_{2}$ must be among $\left\{d, d^{\prime}\right\}$; it follows that $e=\left(l_{2}+l_{3}\right) / 2$ must hold, and the two possibilities are isomorphic.

012. Let $u$ be such that $G_{u}$ has 1 1-limb and a limb of size $\geq 2, x$ being the root of the latter. This case differs from case 011 only in that either the limb at $x$ is of size $\geq 3$, or there is an isolated vertex.

111. Let $u$ be such that $G_{u}$ has 2 1-limb and a 1 limb of size $\geq 2, x$ being the root of the latter. Let $e, e^{\prime}$ be the circular distance from the roots $r, r^{\prime}$ of the 1-limbs of $G_{u}$ to $x$, along the arc not containing the other 1-limb. For a possibility for $y$ to be allowed, it must lie on the arc between $r$ and $r^{\prime}$, not containing $x$. If $e=e^{\prime}$ the possibilities yield isomorphic graphs. If either $P_{2}$ or $P_{3}$ is symmetric the degree 1 vertex of its 1 -limb is determining. Otherwise an ambiguity can occur only if $l_{3}-e=e^{\prime}$ and $l_{2}-e^{\prime}=e$, whence $l_{2}=l_{3}$ and $y$ is determined.

112. Let $u$ be such that $G_{u}$ has 2 1-limbs and a 1 limb of size $\geq 2, x$ being the root of the latter. This case differs from case 111 only as case 012 differs from case 011 .

122. Let $u$ be such that $G_{u}$ has 31 -limbs and a 1 limb of size $\geq 2$, or 1 1-limb, 1 limb of size 2 , and 1 limb of size $\geq 3, x$ being the root of the largest. In the latter case the size 2 path is readily located on the cycle. In the former let $P_{2}$ be the L-path with 2 1-limbs and $P_{3}$ the L-path with 1 1-limb. Write $l_{2}=b_{1}+b_{2}+b_{3}$ where $b_{1}, b_{3}$ are the distances from the roots of the 1-limbs to 
the ends and $b_{2}$ is the distance between them. Similarly write $l_{3}=a_{1}+a_{2}$. One direction of traversing the cycle may be written (renumbering as necessary) as $a_{1}, a_{2}+b_{1}, b_{2}, b_{3}$. An ambiguity can occur only if this sequence equals one of 4 possibilities with the $b$ 's first. These may be seen to all be impossible.

123. Let $P_{1}$ be as in case 122 ; the argument differs from case 122 only in how $P_{1}$ is determined. It may be assumed that there is at most one isolated vertex in $G_{u}$.

\section{L-Multipaths}

An L-multipath in an SBT graph $G$ is defined to be the subgraph consisting of all L-paths between a pair of E-vertices. The number of L-paths is called the multiplicity. The size of an L-multipath is the sum of the sizes of its limbs.

If $G$ is a graph with no degree 1 vertices let $\operatorname{MPG}(G)$ (the "multipath graph" of $G$ ) be the graph whose vertices are the E-vertices of $G$; two such are joined by an edge iff they are joined by one or more T-paths in $G$. If $B$ is an inseparable graph, $\mathrm{MPG}(B)$ equals $\mathrm{MPG}\left(B_{r}\right)$. If $B$ is an S-minimal inseparable graph then $\mathrm{MPG}(B)$ is obtained from $B$ by deleting all T-paths of length 2 . For an inseparable graph $B$, write $B_{m}$ for $\operatorname{MPG}(B) . B_{m}$ is inseparable, and any inseparable graph may occur as $B_{m}$ (replace each edge by a triangle, or add 2 length 2 paths to an edge).

Note that an automorphism of $B$ acts on $B_{m}$.

For an inseparable graph $B B_{m}$ is an edge iff $n_{\bar{v}}^{\geq 3}(B)=2$. Otherwise $B_{m}$ has no degree 1 vertices, else $B$ would be separable. When $n_{p}=1 n_{\bar{v}}^{\geq 3}(B)=2$ and $B_{m}$ is an edge. By theorem $8, G$ is reconstructible in this case.

An L-multipath may be oriented by labeling the vertices of each T-path consecutively starting at an E-vertex. If $M$ is an oriented L-multipath let $M^{r}$ denote $M$ with the opposite orientation; and for a degree 1 vertex $v$ of a limb let $M_{v}$ have the inherited orientation.

Theorem 9. Suppose $M$ is an oriented L-multipath of size $s \geq 2$, and for every degree 1 vertex $v$ of a limb, $M_{v}$ is an oriented subgraph of $M^{r}$. Then $M=M^{r}$.

Proof. If there is only one path with limbs the claim follows by theorem 4; suppose there is more than 1 . Let $m$ be the smallest nonzero size of an L-path. Let $P_{j}$ for $1 \leq j \leq k$ be the L-paths. Let $v$ be a degree 1 vertex of a limb on a 
path $P_{i}$ of size $m$. Let $\pi$ be a permutation of $[1, k]$ such that $P_{j v} \subseteq P_{\pi(j)}^{r}$ for all $j$. Let $S$ be the indices of the L-paths of size $>m$; then $\pi[S]=S$. Thus, for any $j \in S$ there is a cycle $j, \pi(j), \cdots, \pi^{t-1}(j), \pi^{t}(j)=j$ such that $P_{\pi^{s}(j)} \subseteq P_{\pi^{s+1}(j)}^{r}$ for $1 \leq s<t$. It follows that $P_{\pi^{s}(j)}=P_{\pi^{s+1}(j)}^{r}$ for $1 \leq s<t$, and so all $P_{\pi^{s}(j)}$ are equal, or $t$ is even and $P_{\pi^{s}(j)}$ equals $P_{j}$ for $t / 2$ values of $s$, and $P_{j}^{r}$ for $t / 2$ values of $s$. In either case, the multiset of L-paths of size $>m$ is symmetric.

Let $T$ be the indices of the size $m$ L-paths. Renumbering, $v$ is in $P_{0}$ and one of the cycles of $\pi$ is $0,1, \ldots, t-1$. The L-path multiset of each other cycle is symmetric. In the 0 cycle, $P_{0}$ appears $\lceil(t+1) / 2\rceil$ times and $P_{0}^{r}$ appears $\lfloor(t+1) / 2\rfloor$ times. If $t$ is even or $P_{0}$ is symmetric then $M$ is symmetric. If all L-paths have size $m P_{0}$ must be symmetric.

If there is an L-path of size $\geq m+2$, let $v$ be a vertex of such. Let $\pi$ be the permutation for $G_{v}$. Then $\pi[T]=T$, so the L-path multiset for $T$ is symmetric, so $M$ is symmetric. In the remaining case there is an L-path of size $m+1$, and for any such $Q$, and any vertex $v$ of a limb of $Q, Q_{v}=P_{0}^{r}$; this is clearly impossible.

Theorem 10. Let $G$ be an SBT graph with $n_{v}^{E}(B) \geq 3$.

a. If $G$ has limbs the L-multipath sizes are determined.

b. If $G$ has more than one $L$-multipath of nonzero size then the L-multipaths are determined.

Suppose the L-multipaths have been determined.

c. If there is an L-multipath of size $s \geq 2$ but none of size $s-1, G$ is determined.

d. For each multiplicity $m$, if there is an L-multipath of multiplicity $m$ and size $s \geq 2$, but none of multiplicity $m$ and size $s-1$, then $G$ is determined.

Proof. Part a is proved similarly to the claim for L-paths in theorem 8, Indeed, the argument for the L-multipath sizes is virtually identical, except for the case of 21 -limbs. Let $x, y$ be the degree 1 vertices. If the multipaths with an I-limb attached in $G_{x}$ or $G_{y}$ differ, or if they are both a path of length 2, then there are 2 size 1 L-multipaths. If they are a path of length $\geq 3$, there is a single size 2 L-multipath iff there is a vertex $w$ such that in $G_{w}$ there is a single limb. If they are a multipath of multiplicity $\geq 2$, there is a single size 2 L-multipath iff there is a vertex $w$ on a path of a multipath of multiplicity $\geq 2$, such that there is an L-multipath with 2 I-limbs of size 1.

The argument for part $\mathrm{b}$ is readily adapted from an argument in the proof of theorem 8.

For part c, let $v$ range over degree 1 vertices of limbs of the L-multipaths 
of size $s$. For each $v$ both the L-multipath $M$ containing $v$, and $M_{v}$, are determined. If for some $v M_{v}$ is not a subgraph of $M^{r}, M$ may be laid over $M_{v}$. Otherwise, by theorem 9 , for any $v, M_{v}$ may be replaced by $M$.

Part $d$ follows as part c, letting $v$ range over degree 1 vertices of L-multipaths of multiplicity $m$ and size $s$.

\section{7. $B_{m}$ a Cycle}

Lemma 11. Suppose $G$ is an inseparable graph. It is determined whether $B_{m}$ is a cycle.

Proof. There must be $\geq 3$ E-vertices. $B_{m}$ is a cycle iff, for any E-vertex $v$, $\operatorname{MPG}\left(G_{v}^{T}\right)$ is a path, where for a graph $G G^{T}$ denotes the trunk of $G$.

Suppose for the rest of the section that $G$ is an SBT graph with $B_{m}$ a cycle. Say that a multipath is fat (resp. thin) if it has multiplicity $>1$ (resp. 1 ). Say that it is $\mathrm{T}_{i}$ if it is thin and has length $i$; and $\mathrm{F}_{i j}$ for $1 \leq i \leq j$ if it has multiplicity 2 and path lengths $i, j$. The same terminology applies to L-multipaths.

The automorphism group of $B$ is either trivial, generated by a rotation, generated by a reflection, or generated by a reflection and a rotation. In cases 2 and 4 say that $B$ admits a rotation. If $B$ admits a rotation then there is a subgraph $Q$ of $B$, which is a concatenation of $q$ multipaths, such that $B$ is the circular concatenation of $q^{\prime}$ copies of $Q$. Further, if $q$ is as small as possible then for any $Q$, these are the only copies of $Q$ around $B$.

Lemma 12. If $G$ has no limbs then $G$ is reconstructible.

Proof. It may be assumed that all T-paths have length at most 3 , since if $v$ is a degree 2 vertex of distance $\geq 2$ from both ends then $G$ may be reconstructed from $G_{v}$.

If there are any $\mathrm{T}_{i}$ multipaths for $i=2,3$ let $w$ be an interior vertex of such; $G$ is determined from $G_{w}$, except that an end cycle of $G_{w}$ of length 4 might be either an $\mathrm{F}_{13}$ or $\mathrm{F}_{22}$ multipath. It is determined whether a vertex $v$ is an interior vertex of the path of length 3 of an $\mathrm{F}_{13}$ multipath, and so the number of $\mathrm{F}_{13}$ multipaths is determined. It follows that if $w$ is an interior vertex of a $\mathrm{T}_{3}$ multipath then $G$ is determined from $G_{w}$. 
Suppose there are no $\mathrm{T}_{3}$ multipaths but there are $\mathrm{T}_{2}$ multipaths. Let $w$ be the interior vertex of a $\mathrm{T}_{2}$ multipath $P$. Unless one end of $P$ belongs to an $\mathrm{F}_{13}$ multipath and the other to an $\mathrm{F}_{22}$ multipath, $G$ is determined from $G_{w}$. In particular it may be assumed that there is a degree 3 vertex $v$ such that in $G_{v}$, one end is a 3 vertex complete binary tree and the other is a path of length 1 ; $G$ may be reconstructed from $G_{v}$.

Suppose there are no $\mathrm{T}_{2}$ or $\mathrm{T}_{3}$ multipaths, and there are $\mathrm{T}_{1}$ multipaths. $\mathrm{A}$ vertex $v$ is the end vertex of a $\mathrm{T}_{1}$ path iff one end $D$ of $G_{v}$ is a fat multipath or a cycle; let $E$ be the other end, which is a tree. The multipath from which $E$ is derived may be determined. This is clear if $E$ is not a path, or if $E$ is a path of length 1 or 3 . If $E$ is a path of length 2 the choice between a $T_{1}$ multipath followed by an $\mathrm{F}_{12}$ multipath, and an $\mathrm{F}_{13}$ multipath, may be made on the basis of the number of $\mathrm{T}_{1}$ multipaths. If $D$ is not a cycle of length $4 G$ is readily determined. If $D$ is a cycle of length $4 G$ is determined also, since the number of $\mathrm{F}_{13}$ multipaths is known.

Letting $v$ be the other end of the $\mathrm{T}_{1}$ multipath, $E$ must be an $\mathrm{F}_{13}$ also. Thus, every $\mathrm{T}_{1}$ multipath must have its ends in an $\mathrm{F}_{13}$ multipath, and so $G$ may be reconstructed from $G_{v}$ where $v$ is an end of a $\mathrm{T}_{1}$ multipath.

Suppose all multipaths are fat. Let $v$ be an E-vertex. Add a new vertex to $G_{v}$ and connect it to the degree 1 vertices. If either 0 or 2 additional edges are required, these may be added as needed to reconstruct $G$ from $G_{v}$. Otherwise, in every adjacent pair of multipaths, one contains a length 1 path and the other does not. Which root of the trees in $G_{v}$ to connect to the new vertex is readily determined in $G_{v}$.

Lemma 13. Let $q$ be the number of limbs of size $\geq 2$. If $q \geq 2$ then $G$ is reconstructible.

Proof. Let $u$ be a degree 1 vertex of a 1 -limb $T$, and let $v$ be a degree 1 vertex of a limb of size $\geq 2$. Let $R$ be $B$ with the limb roots marked, and let $R_{u}$ be $B$ with the roots marked, other than that of $T$.

The cases where $R$ has a rotation which is an automorphism, and where $R$ is rigid, may be proved as in the case of lemma 5 .

In the remaining case, the automorphism group of $R$ is generated by a single reflection, and the axis may be determined in any $G_{v}$. $G$ may be divided into two subgraphs $P_{1}$ and $P_{2}$, each containing the vertices on one side of the axis, where a vertex on the axis and its attached limb if any are in both $P_{i}$.

If $q \geq 3$ then $P_{1}$ and $P_{2}$ in $G$ may be determined by considering the $G_{v}$, as in the proof of lemma 5 . 
Suppose $q=2$. The roots $r_{1}$ and $r_{2}$ of the size $\geq 2$ limbs may be located in $P_{1}$, so it only remains to determine if in $G$ they are located in opposite sides.

If $r_{1}=r_{2}$ then they are on opposite sides. If one of $r_{1}, r_{2}$ is on the axis the question is irrelevant. If one of the limbs (say the one at $r_{1}$ ) has size 3 then this can be noted in the marked positions in $P_{1}$; whether $r_{1}, r_{2}$ are on the same side can then be determined from $G_{v}$ where $v$ is a degree 1 vertex of the limb of size 3 .

Suppose $r_{1}, r_{2}$ are in the same L-multipath $M$ in $P_{1}$. It may be determined whether they are in the same multipath in $G$ from any $G_{u}$. Unless $M$ is on the axis, $G$ may clearly be reconstructed. If $M$ is on the axis, the axis is that of $M$ with its limbs removed, so whether $r_{1}, r_{2}$ are on the same side is determined from any $G_{u}$.

Suppose there is no L-multipath containing $r_{1}, r_{2}$.

Suppose there are L-multipaths $M_{1}, M_{2}$ on the axis with $r_{1}$ in $M_{1}$ and $r_{2}$ in $M_{2}$. If either limb is an I-limb, the axis is known. Otherwise, the distance $d$ between $r_{1}$ and $r_{2}$ in $B_{m}$ can be determined from any $G_{u} ; r_{1}, r_{2}$ are on opposite sides iff $d=l / 2$ where $l$ is the length of $B_{m}$.

Suppose $r_{1}$ is on $M_{1}$ where $M_{1}$ is on the axis, but $r_{2}$ is not on the other L-multipath on the axis, if any. In $G_{u}$, let $w$ be the E-vertex of $M_{1}$ closest to $r_{2}$, and let $P$ be the T-path of $M_{1}$ containing $r_{2}$, and let $l$ be its length. Then $r_{1}$ and $r_{2}$ are on the same side iff the distance from $r_{1}$ to $w$ is less than $l / 2$.

In the remaining case, let $C$ be a cycle of length $2 l$, with alternate positions denoting E-vertices and L-multipaths. Number the nodes counterclockwise from 0 , where node 0 is an E-vertex or L-multipath according to which lies at the top of the axis. The locations of $r_{1}$ and $r_{2}$ on one side of $C$ are determined; let these be $0<i<j<l$. If $r_{1}, r_{2}$ are on the same side the distance between them in $C$ is $j-i$. If they are on opposite sides, if $i+j \leq l$ the distance is $i+j$, and if $i+j>l$ the distance is $2 l-i-j$; in either case it may be determined if $r_{1}, r_{2}$ are on the same side.

In the remaining cases, $B$ is known.

Lemma 14. Suppose $G$ has 1 limb; then $G$ is reconstructible.

Proof. By theorem 2 the limb may be assumed to be a 1-limb. Let $x$ be its root.

Suppose the limb is an I-limb. The L-multipath $M$ containing $x$ may be determined from $G_{x}$. Indeed, the multipath is known from $B$, and the location of $x$ may be determined from the limbs of $G_{x}$. 
Suppose there is a thin L-multipath $P$ of length $\geq 4$. Let $z$ be an interior vertex of $P$ which is at distance 2 from an end whose neighbor interior vertex has no limb. $G$ may be readily reconstructed from $G_{z}$, no matter where $x$ is located.

If $M$ is a $\mathrm{T}_{3}$ L-multipath there is a vertex $y$ such that in $G_{y}$ one end $E_{1}$ is a multipath with a path of length 2 attached, and the other end $E_{2}$ is a multipath or cycle. The multipath $M_{1}$ and $M_{2}$ giving rise to $E_{1}$ and $E_{2}$ may be determined, and $G$ reconstructed from $G_{y}$.

If $M$ is a $\mathrm{T}_{2}$ L-multipath the ends $E_{1}$ and $E_{2}$ in $G_{x}$ are each a multipath or cycle. Unless both are 4-cycles, $G$ is readily reconstructed; this is also true if the multipaths missing from $B$ are both $\mathrm{F}_{22}$ or $\mathrm{F}_{13}$ multipaths. In the remaining case there is a vertex $y$ such that in $G_{y}$ there is a path of length $\geq 4$, with an $\mathrm{F}_{13}$ multipath at one end with a 1-limb attached to the closest vertex to that end of the path; $G$ is readily reconstructed from $G_{y}$.

In the remaining cases $M$ is a fat multipath.

If $M$ is adjacent to a $\mathrm{T}_{3} \mathrm{~L}$-multipath there is a vertex $y$ such that in $G_{y}$ one end $E_{1}$ is a cycle or multipath with 2 1-limbs attached, and the other end $E_{2}$ is a cycle or multipath. $E_{1}$ is derived from $M$, so the multipath from which $E_{2}$ is derived is also known. If $E_{1}$ is a multipath $G$ is readily reconstructed from $G_{u}$. Otherwise $M$ is an $F_{i j}$ L-multipath. If $i=j G$ is readily reconstructed, so suppose $i<j$. Let $l_{1} \leq l_{2}$ be the distances from the attachment vertex of $E_{1}$ to the roots of the limbs; if $l_{1}=l_{2} G$ is readily reconstructed, so suppose $l_{1}<l_{2}$. It is readily verified that if the 1-limb is on the path of length $i$ then $j=l_{2}$, and if it is on the path of length $j$ then $i=l_{1} ; G$ may thus be reconstructed.

If $M$ is adjacent to a $\mathrm{T}_{2}$ L-multipath there is a vertex $y$ such that in $G_{y}$ one end $E_{1}$ is a cycle or multipath with a 1 -limb attached, and the other end $E_{2}$ is a cycle or multipath. $E_{1}$ may be replaced by $M$. The multipath missing from $B$ is then known, and $G$ may be reconstructed.

If $M$ is adjacent to a $\mathrm{T}_{1} \mathrm{~L}$-multipath there is a vertex $y$ such that in $G_{y}$ one end $E_{1}$ is a cycle or multipath with a 1-limb attached, and the other end $E_{2}$ is a tree. $E_{1}$ may be replaced by $M$. The multipath $M_{2}$ missing from $B$ may then be determined, and also the part of it to be replaced may be determined and $G$ reconstructed.

Suppose there is a vertex $y$ such that in $G_{y}$ there is an L-multipath with an I-limb and an E-limb (both 1-limbs). Since the missing multipath is known from $B$, a new vertex may be connected to the end of the E-limb, and to a second vertex, to reconstruct $G$ from $G_{y}$.

If there is no such $y$, let $w$ be such that in $G_{w}$ the number of length 2 paths in a multipath adjacent to $M$ is as small as possible; $G$ may be reconstructed 
from $G_{w}$.

Now suppose that the limb is an E-limb, with root $v$. At least 1 multipath $M$ incident to $v$ is fat. If the other $T$ is thin, the argument is similar to arguments already given. Namely, if $T$ has length $\geq 4$ let $u$ on $T$ have distance at least 2 from both ends; if $T$ has length 3 let $u$ such that $E_{1}$ in $G_{u}$ has a limb of size 2 ; if $T$ has length 2 let $u$ be such that $E_{1}$ has a 1 limb; and if $T$ has length 1 let $u$ be such that $E_{1}$ has a 1-limb.

In the remaining case, the other multipath $N$ incident to $v$ is also fat. If one of $M, N$ has a path of length 3, suppose w.l.g. that $M$ is one of smallest multiplicity. Let $u$ be on $M$ such that in $G_{u}$ there is a single limb, of size $\geq 2$. Unless $M_{u}=N G$ may be reconstructed from $G_{u}$. If $N=M_{u} G$ is reconstructible from $G_{w}$ where $w$ is on a length 2 path of $M$. If neither $M, N$ has a path of length 3 suppose w.l.g. that $M$ has smallest multiplicity between $M, N ; G$ is reconstructible from $G_{w}$ where $w$ is on a length 2 path of $M$.

Lemma 15. Suppose $B$ admits a rotation; then $G$ is reconstructible.

Proof. Let $x$ be a vertex on an L-path of an L-multipath $M$ of multiplicity $\geq 2$ in $G$. With $q$ as at the start of the section, let $Q$ be a run of $q$ multipaths, not including the multipath $M_{0}$ missing from $G_{x}$. Using $Q$, the location of $M_{x}$ in $G_{x}$ may be found. $x$ may be chosen so that the E-limbs of $G_{x}$ are those of $G$, with one of them having an extra subtree $A$ of its root; further the root is an E-vertex of $M$.

$G$ may be reconstructed from $G_{x}$ by adding a new vertex $x$, connecting it to the E-vertex of $M_{x}$ other than the root of $A$, connecting it as necessary to any disconnected component(s), and connecting it to the correct vertex of $A$. The latter may be determined, since the length of the L-path containing $x$ is known, and I-limbs are 1-limbs, with the possible exception of a single I-limb of size 2 .

Lemma 16. Suppose the automorphism group of $B$ is generated by a reflection; then $G$ is reconstructible.

Proof. Similarly to the proof of lemma 13 when the automorphism group of $R$ is generated by a single reflection, the axis may be determined in any $G_{u}$ where $u$ is a degree 1 vertex. $G$ may be divided into two parts $P_{1}$ and $P_{2}$. If the number $q$ of limbs is $\geq 3 G$ may be reconstructed as in lemma 13 .

Suppose $q=2$. The roots $r_{1}$ and $r_{2}$ of the limbs may be located in $P_{1}$, so it only remains to determine if in $G$ they are located in opposite sides. 
If $r_{1}=r_{2}$ then they are on opposite side. If one of $r_{1}, r_{2}$ is on the axis the question is irrelevant. If one of the limbs (say the one at $r_{1}$ ) has size 2 then this can be noted in the marked positions in $P_{1}$; whether $r_{1}, r_{2}$ are on the same side can then be determined from $G_{v}$ where $v$ is a degree 1 vertex of the limb of size 2 .

Suppose $r_{1}, r_{2}$ are in the same L-multipath $M$ in $P_{1}$. Suppose $M$ is thin. If $M$ is on the axis there is a vertex $w$ on the path of $M$ such that in $G_{w}$ there is only one limb, and $G$ may be reconstructed from $G_{w}$. If $M$ is not on the axis there is a vertex $w$ on the path of $M$ such that in $G_{w}$ there is only one limb, iff $r_{1}, r_{2}$ are on the same side. Suppose $M$ is fat. If $M$ is on the axis let $w$ be the root of an I-limb of $M ; G$ may be reconstructed from $G_{w}$. If $M$ is not on the axis let $w$ be the root of an I-limb of $M$ if there is one, else any vertex on a T-path of $M$; it can be determined from $G_{w}$ whether $r_{1}, r_{2}$ are on the same side.

Suppose there is no L-multipath containing $r_{1}, r_{2}$. Let $C$ be the cycle of length $2 l$ as in the proof of lemma 13; it suffices to determine the distance in $C$ between the nodes corresponding to $r_{1}, r_{2}$.

Suppose $r_{1}, r_{2}$ are I-vertices of L-multipaths $M_{1}, M_{2}$. If there is a fat multipaths $M_{3}$ distinct from these let $w$ be a vertex on a path of $M_{3}$; $d$ may be determined from $G_{w}$. Otherwise $l=3$ and one l-multipath is thin; all possibilities have already been covered.

Suppose $r_{1}$ is an E-vertex with a 1-limb attached, with degree 1 vertex $u$. If $l=3 G$ may be reconstructed from $G_{u}$, since $r_{2}$ must be an Suppose $l=4$. If there is an E-vertex on the axis, or if $r_{2}$ is an E-vertex, $G$ is reconstructible from $G_{u}$. Otherwise whether the $r_{2}$ L-multipath is parallel or perpendicular to the axis is known from $G_{u}$, and $G$ is reconstructible from $G_{t}$ where $t$ is the other degree 1 vertex.

Thus, it may be assumed that $l \geq 5$. Let $l_{1}$ be the number of L-multipaths in $G_{r_{1}}$, including the end multipaths or cycles, which will be denoted $M_{1}, M_{2}$ $\left(M_{1}=M_{2}\right.$ iff $\left.l=5\right)$. Let $l_{2}=l_{l}-1$, so that $2 \leq l_{2} \leq 4$. Let $v_{1}, v_{2}$ be the E-vertices of $M_{1}, M_{2}$ in $G_{r_{1}}$ (these exists iff $l \geq 6$ and are unequal iff $l \geq 7$, and say that $P\left(r_{2}\right)$ holds if $r_{2}$ is $v_{1}, v_{2}$, or lies between them; if this is the case let $d_{i}$ be the distance from $r_{2}$ to $v_{i}, i=1,2$. Let $j$ be the number of fat multipaths incident to $r_{1}$.

The rest of the proof is broken into the cases $l_{2} j$.

Case 42. If $P\left(r_{2}\right)$ then $d=\min \left(d_{1}, d_{2}\right)+6$. Otherwise, $d=5$ if $r_{2}$ is an I-vertex of a fat multipath, $d=4$ if $r_{2}$ is an E-vertex, and $d=3$ if $r_{2}$ is an I-vertex of a thin multipath.

Case 32. W.l.g. the L-multipaths from $M_{1}$ to $M_{2}$ may be written $M_{1} F_{1} F_{2} T_{2} M_{2}$ 
where $F_{i}$ is fat and $\mathrm{T}_{2}$ is thin. Unless for some $p \geq 2, q \geq 1 F_{1}$ is $\mathrm{F}_{1, p+q}, F_{2}$ is $\mathrm{F}_{1, p}$, and $T_{2}$ is $\mathrm{T}_{q}, M_{1}$ is known in $G_{r_{1}}$; suppose this is the case. If $P\left(r_{2}\right)$ then $d=\min \left(d_{1}+4, d_{2}+6\right)$. Otherwise, if $r_{2}$ is an I-vertex of a thin multipath then $d=3$, if $r_{2}$ is an E-vertex then $d=4$, and if $r_{2}$ is an I-vertex of a fat multipath then $d=3$ if $M_{1}$ has 2 limbs, else $d=5$. In the remaining case there is a vertex $w$ such that in $G_{w}$ there is an E-vertex with attached branches of size 1 and $p+q-2$, with missing $\mathrm{F}_{1, p+q}$ multipath, etc.; $G$ is reconstructible from $G_{w}$.

Case 22. If $P\left(r_{2}\right)$ then $d=\min \left(d_{1}, d_{2}\right)+4$. Otherwise, $d=3$.

Case 31. W.l.g. the L-multipaths from $M_{1}$ to $M_{2}$ may be written $M_{1} T_{1} F_{2} T_{2} M_{2}$ where $F_{2}$ is fat and $\mathrm{T}_{i}$ is thin. Unless for some $p \geq 2, q \geq 1 T_{1}$ is $\mathrm{T}_{p+q}, F_{2}$ is $\mathrm{F}_{1, p}$, and $T_{2}$ is $\mathrm{T}_{q}, M_{1}$ is known in $G_{r_{1}}$; suppose this is the case. If $P\left(r_{2}\right)$ then $d=\min \left(d_{1}+4, d_{2}+6\right)$. Otherwise, if $r_{2}$ is an I-vertex of a thin multipath then $d=3$, if $r_{2}$ is an E-vertex then $d=4$, and if $r_{2}$ is an I-vertex of a fat multipath then $d=5$ if $M_{2}$ has 2 limbs, else $d=3$. In the remaining case there is a vertex $w$ such that in $G_{w}$ there is an end multipath or cycle with a vertex with attached branches of size 1 and $p+q-2$, with missing $\mathrm{T}_{p+q}$ multipath, etc.; $G$ is reconstructible from $G_{w}$.

Case 21. Same as case 22.

Theorem 17. If $B_{m}$ is a cycle then $G$ is reconstructible.

Proof. This follows by lemmas 12 to 16 , and theorem 3 .

\section{8. $B_{m} K_{4}$}

In this section, $G$ will denote an SBT graph with $B_{m}$ equaling $K_{4}$. In two cases, a fact is shown for all but a finite number of graphs, and the fact verified for them by a computer program. The "Nauty" library [5] was used for graph canonicalization in these cases.

Lemma 18. If $G$ has no limbs then $G$ is reconstructible.

Proof. If for some $m \geq 2$ there is a multipath of multiplicity $m$ but none of multiplicity $m-1$, let $v$ be a vertex on a path of such a multipath; then $G$ is reconstructible from $G_{v}$. Thus, the multipath multiplicities may be assumed to form an interval $[1, m]$. 
Suppose all thin multipaths are edges, and there is at least 1 fat multipath. Let $m=2$. If a multipath $M$ of multiplicity $m$ has all paths of length $\geq$ 2 let $v$ be an interior vertex of a path of $M ; G$ is reconstructible from $G_{v}$. Proceeding inductively on $m$ and using the same argument, every fat multipath must contain an edge. It now follows that $G$ is reconstructible from $G_{v}$ where $v$ is any E-vertex.

Suppose there is a thin multipath $P$ of length $\geq 2$ and let $v$ be an interior vertex. Unless for one of the end vertices of $P$ both other incident multipaths are thin, $G$ may be reconstructed from $G_{v}$. Thus, there must be an E-vertex $w$ with all 3 incident multipaths thin. If in the triangle of remaining multipaths two of them are fat then the E-vertices in $G_{v}$ may be found and $G$ reconstructed.

Suppose there is a fat multipath $M$ and 5 thin multipaths, at least one of which has length $\geq 2$. If the multipath opposite $M$ has length $\geq 2$ then $G$ is reconstructible from $G_{w}$, else $G$ is reconstructible from $G_{v}$, where $v$ and $w$ are as above.

Suppose all multipaths are thin. Let $q$ be the number of multipaths which are edges; this is half the sum of the numbers at the E-vertices. If $q \geq 5$ there is a triangle of edges. If there is a triangle of edges let $v$ be the E-vertex not on it; then $G$ is reconstructible from $G_{v}$.

Suppose it is known that there is a parallel pair of edges; $G$ is reconstructible from $G_{v}$ where $v$ is an interior vertex of a thin multipath of length $\geq 2$. If $3 \leq q \leq 4$ and there is no triangle then there is a parallel pair of edges.

Say that an E-vertex is connectible if the 3 incident thin multipaths have length $\geq 2$; if $v$ is a connectible E-vertex then $G$ is reconstructible from $G_{v}$. If $q \leq 1$ or $q=2$ and the edges are adjacent then there is a connectible Evertex.

For the rest of the section it will be assumed that $G$ has limbs. If $i, j$ are E-vertices call the L-multipath joining $i$ and $j$ the $i j$ multipath.

Lemma 19. If $G$ has more than 1 E-limb then $G$ is reconstructible.

Proof. Let $G^{I}$ be $G$, with the E-limbs removed; $G^{I}$ is known from $G_{u}$ where $u$ is a degree 1 vertex of an E-limb. Consider the orbits of the automorphism group of $G^{I}$.

By a typical argument, for each orbit the E-limbs attached to the nodes of the orbit may be determined. Let $r$ be the number of E-limbs of size 1; then either $r>1$, or $r=1$ and there are E-limbs of size $>1$. The E-limbs of size $>1$ belonging to each orbit may be determined from $G_{u}$ where $u$ is the degree 1 
vertex of a size $1 \mathrm{E}$-limb. If $r>1$ let $u$ range over the degree 1 vertices of size 1 E-limbs; the orbits containing size 1 E-limbs may be determined, and then the number in each such orbit, from the $G_{u}$. If $r=1$ let $u$ be a degree 1 vertex of an E-limb of size 2; the orbit containing the size 1 E-limb may be determined from $G_{u}$.

The proof of the lemma is divided into cases, according to the orbit size list.

Case 1111. The vertex to which each E-limb is attached is known.

Case 112. The automorphism group acts transitively on the size 2 orbit, so the trees assigned to it may be assigned to the nodes of the orbit arbitrarily.

Case 22. Both orbits may be assumed to have a single E-limb, of size 1, since otherwise $G$ can be reconstructed from the $G_{u}$ where $u$ is a degree 1 vertex of a size 1 E-limb. If the automorphism group is $Z_{2} \times Z_{2}$ (with generators (01),(23), say), the trees assigned to each orbit may be assigned to the nodes of the orbit arbitrarily.

Suppose the automorphism group is $Z_{2}$, w.l.g. with generator $(01)(23)$. The 01 L-multipath $A$ and the 23 L-multipath $A^{\prime}$ are symmetric. Let $B$ denote the 02 and 13 L-multipaths and $C$ the 03 and 12. $B \neq C$, else the automorphism group is not $Z_{2}$.

If at least 1 of $B, C$ (w.l.g. $B$ ) is asymmetric let $u$ be a degree 1 vertex of an I-limb. In $G_{u}$ there is a unique copy of $B$, and it is readily determined whether the E-limbs are at the ends of a copy of $B$, and $G$ may be reconstructed from $G^{I}$. If $B, C$ are symmetric then $A \neq A^{\prime}$. If at least 1 of $B, C$ (say $B$ ) has I-limbs let $u$ be a degree 1 vertex of an I-limb. The pair of $C$ L-multipaths may be found in $G_{u}$ and it may be determined whether the E-limbs are at the ends of a copy of $C$ and $G$ may be reconstructed from $G^{I}$. The argument if either $A$ or $A^{\prime}$ has an I-limb is similar.

If at least one of $B, C$ is fat (say $B$ ) let $w$ be a vertex on a path of $B$ such that $G_{w}$ has only 2 limbs. The two parallel copies of $C$ may be found in $G_{w}$ and it may be determined whether the E-limbs are at the ends of a copy of $C$. The argument if either $A$ or $A^{\prime}$ is fat is similar.

In the remaining case let $w$ be a vertex on the longer of $A, A^{\prime}$ such that in $G_{w}$ there are two limbs. It may be determined from $G_{w}$ whether the limbs are at the ends of a copy of $B$ or $C$, and $G$ may be reconstructed from $G_{w}$.

Case 13. If the automorphism group is $S_{3}$ the 1-limbs on the size 3 orbit may be assigned arbitrarily to the nodes of the orbit. Otherwise, numbering so that the size 3 orbit is 012 , there is an asymmetric L-multipath $A$ such that the 01,12 , and 20 L-multipaths all equal $A$. $A$ must have I-limbs, and $G$ is reconstructible from $G_{u}$ where $u$ is a degree 1 vertex of an I-limb if $A$. 
Case 4. If the automorphism group is $Z_{2} \times Z_{2}$ the 01,23 multipaths are both $A$, the 02,13 multipaths are both $B$, and the 03,12 multipaths are both $C$, where $A, B$, and $C$ are symmetric and all distinct. The proof is divided into subcases, according to the E-limb size list $S$. If $S$ is 11 the proof is similar to the previous case of orbit sizes 22 and automorphism group $Z_{2} \times Z_{2}$. If there are any I-limbs let $u$ be a degree 1 vertex of one; then which of $A, B, C$ join the 2 E-limbs may be determined from $G_{u}$ and $G$ reconstructed from $G^{I}$. If there is a fat multipath let $w$ be a vertex on a path of one such that $G_{w}$ has only 2 limbs; which of $A, B, C$ joins them may be determined from $G_{w}$. In the remaining case let $w$ be a vertex along the longest of $A, B, C$ such that $G_{w}$ has 2 limbs,

If $S$ is 12 the L-multipath joining the two E-limbs can be determined from $G_{u}$ where $u$ is a degree 1 vertex of the size 2 E-limb. If $S$ is 111 the E-limbs may be assigned to E-vertices arbitrarily. If $S$ is 112 the two L-multipaths joining the size 2 E-limb to a size 1 E-limb are determined from the $G_{u}$ where $u$ is the degree 1 vertex of a size $1 \mathrm{E}$-limb. If $S$ is 122 the L-multipath joining the 2 size 2 E-limbs can be determined from $G_{u}$ where $u$ is the degree 1 vertex of the size 1 E-limb. If $S$ is 123 the L-multipath joining the size 2 and size 3 E-limb may be determined from $G_{u}$ where $u$ is the degree 1 vertex of the size $1 \mathrm{E}$-limb; and the L-multipath joining the size 1 and size 2 E-limb may be determined from $G_{u}$ where $u$ is a degree 1 vertex of the size 2 E-limb.

If the automorphism group is $Z_{4}$ the nodes may be numbered so that the $01,12,23$, and 30 L-multipaths are all equal to an asymmetric L-multipath $A$. $\mathrm{G}$ is readily reconstructed from $G_{u}$ where $u$ is a degree 1 vertex of an I-limb of $A$.

If the automorphism group is $D_{8}$ the graph consists of 3 parallel classes of L-multipaths, two of which are equal. The argument is essentially the same as in the case of 3 distinct parallel classes given above.

In the remaining case the automorphism group is $S_{4}$ and the E-limbs may be assigned arbitrarily to the E-vertices.

Lemma 20. If $G$ has 1 E-limb and the sum of the sizes of the I-limbs is $\geq 2$ then $G$ is reconstructible.

Proof. Let $v$ denote the E-vertex with the E-limb attached. Let $\Sigma_{1}$ denote the set of L-multipaths incident to $v$, with $v$ marked. Let $\Sigma_{2}$ denote the remaining 3 L-multipaths. For $i=1,2$ let $s_{i}$ denote the sum of the sizes of the limbs of $\Sigma_{i}$; by a standard argument $s_{1}$ and $s_{2}$ may be determined, and then $\Sigma_{1}$ and $\Sigma_{2}$, from the $G_{u}$ where $u$ ranges over the degree 1 vertices of the I-limbs. By an 
argument as in theorem 10 the L-multipath sizes in both $\Sigma_{1}$ and $\Sigma_{2}$ must form an interval starting at 0 . If $s_{1}=0$ then $v$ is the only E-vertex with 3 incident size 0 L-multipaths, else there is only 1 L-multipath of nonzero size and $G$ is reconstructible by theorem 10 . Likewise, $s_{2} \neq 0$ may be assumed.

Let $G^{E}$ be $G$ with I-limbs deleted. If $G^{E}$ is rigid $G$ may be reconstructed by taking the union of the $G_{u}$ where $u$ ranges over degree 1 vertices of I-limbs. Otherwise, number E-vertices so that the E-vertex with the E-limb attached is 0 , and (12) is an automorphism of $G^{E}$.

Suppose the 03 multipath $B$ differs from the 01 and 02 multipaths $A$. If the 12 multipath has any I-limbs then $G$ is reconstructible from $G_{u}$ where $u$ is a degree 1 vertex of one; likewise for the 03 L-multipath. If the 01 and 02 L-multipaths both have nonzero size then $G$ is reconstructible from $G_{u}$ where $u$ is a degree 1 vertex of an I-limb on a copy of $A$ of smallest size. If the 03 and 13 multipaths differ from $B$, likewise for them. If they equal $B$ there is only one vertex in $G^{I}$ with 3 incident equal multipaths, and $G$ is reconstructible from $G^{I}$. Thus, there are 2 nonzero size L-multipaths. From $G^{I}$ it is known whether they are vertex disjoint, and so $G$ is reconstructible from $G_{u}$ where $u$ is the degree 1 vertex of the I-limb on a copy of $A$.

Suppose $B=A$. If there is only 1 E-vertex with 3 incident multipaths equaling $A$ then $G$ is reconstructible from $G^{I}$. Otherwise, all multipaths are $A$, except possibly the 02 multipath. If it differs, similarly to arguments just given, it must be size 0 , whence the 02 multipath must be size 0 , and the argument proceeds as before.

Suppose all 6 multipaths equal $A$. If there are 2 nonzero size L-multipaths in $\Sigma_{2}$, w.l.g. the 12 L-multipath may be assumed to have size 0. As usual the 03 L-multipath has size 0 , and so only one L-multipath in $\Sigma_{1}$ has nonzero size, say the 01 L-multipath. $G$ may be reconstructed from $G_{u}$ where $u$ is a degree 1 vertex of an I-limb of the $13 \mathrm{~L}$-multipath. In the remaining case of two nonzero size multipaths the argument is as before.

Lemma 21. If $G$ has 1 E-limb and a single I-limb of size 1 then $G$ is reconstructible.

Proof. Let $v, \Sigma_{1}, \Sigma_{2}$ be as in the previous proof. Let $A$ be the L-multipath of nonzero size. $A$ is in $\Sigma_{1}$ iff there is a vertex $w$ on a path, such that $G_{w}$ has only 1 limb.

Suppose $A$ is in $\Sigma_{1}$. Let $w$ be the root of the I-limb. From $G_{w}$ the Lmultipaths in $\Sigma_{1}$, with $v$ marked, may be determined, and also the multipath joining the other ends of the two such other than $A$. If $\Sigma_{1}$ contains only 1 copy 
of $A$ then $G$ may be reconstructed from $G_{u}$ where $u$ is the degree 1 vertex of the I-limb. Otherwise, let $w$ be a vertex on a path of a second copy of $A$ such that in $G_{w}$ there is only 2 limbs; $v$ can be located in $G_{w}$, and also the 4 th E-vertex since $B$ is known. Thus, $G$ may be reconstructed from $G_{w}$.

Lemma 22. If $G$ has 1 E-limb and no I-limbs then $G$ is reconstructible.

Proof. The argument in the proof of lemma 18 that $G$ is reconstructible if there is a fat multipath carries through if there is a single E-limb, with some modifications.

Suppose the E-vertices are numbered so that 0 has 3 incident thin multipaths, and the 12 and 23 multipaths are fat. $G$ is reconstructible from $G_{v_{0}}$ unless the vertex with the E-limb attached is (w.l.g.) $v_{1}$ and the 03 multipath has length 2. In this case $G$ is reconstructible from $G_{w}$ where $w$ is the vertex on the 03 path.

Suppose there is a fat multipath $M$ and 5 thin multipaths, at least one of which has length $\geq 2$. The length $l$ of the path opposite $M$ is known since $B$ is. If there is a path $P$ of length $\geq 2$ incident to an E-vertex of $M$ let $w$ be a vertex of $P$ such that in $G_{w}$ there are as few limbs as possible. $G$ is reconstructible from $G_{w}$ since $l$ is known. In the remaining case the paths incident to the E-vertices of $M$ are edges, and the remaining path $P$ has length $\geq 2$. $G$ may be reconstructed from $G_{w}$ where $w$ is an interior vertex of $P$ such that in $G_{w}$ the number of limbs is as small as possible.

Suppose all multipaths are thin. Let $v, \Sigma_{1}, \Sigma_{2}$ be as in the previous proof. $\Sigma_{1}$, and hence $\Sigma_{2}$, are known from $G_{v}$. If all paths in $\Sigma_{1}$ have length 1 then $G$ is reconstructible because $\Sigma_{2}$ is known. If some path in $\Sigma_{1}$ has length $\geq 4$ then $G$ is reconstructible from $G_{w}$ where $w$ is on such a path, at distance $\geq 2$ from each end. If some path in $\Sigma_{2}$ has length $\geq 6$ then $G$ is reconstructible from $G_{w}$ where $w$ is on such a path, at distance $\geq 3$ from each end. There remain only a finite number of graphs; a computer program was written to verify that these all have distinct decks.

Lemma 23. If $G$ has a fat $L$-multipath then the $L$-multipaths are determined.

Proof. This is trivial if $G$ has an E-limb; suppose not. By theorem 10 it suffices to consider the case of a single L-multipath $M$ of nonzero size. If $M$ is thin let $v$ be a vertex on a path of a fat multipath. If $M$ is fat let $v$ be an E-vertex not incident to $M$. In either case $M$ is an L-multipath of $G_{v}$. 
Lemma 24. Suppose $P$ is an $L$-path of length $\geq 2$ with its ends marked and $v$ is a vertex on $P$. Then either $P$ lies over $P_{v}$ in only one way, or $P$ is symmetric.

Proof. Orient $P$ and let $T_{1}, \ldots, T_{l-1}$ be the trees along $P$, where $l$ is the length of $P$. Say that $P$ can proceed along $P_{v}$ to $i$ if it can proceed to $i-1$ (vacuous if $i=1$ ), and a copy of $T_{i}$ can be cut out of the remaining subtree of $P$. It is readily verified that if $P$ in both orientations can proceed along one of the two trees of $P_{v}$ of maximum length to $\lfloor(l-1) / 2\rfloor$ then $P$ is symmetric, proving the lemma.

Lemma 25. If $G$ has no E-limbs and at least 1 fat $L$-multipath then $G$ is reconstructible.

Proof. The proof is a modification of statements in the proof lemma 18. If for some $m \geq 2$ there is a multipath of multiplicity $m$ but none of multiplicity $m-1$, let $v$ be a vertex on a path of such a multipath. Using lemmas 23 and $24, G$ is reconstructible from $G_{v}$. Thus, the multipath multiplicities may be assumed to form an interval $[1, m]$.

Suppose there is a thin L-multipath $P$ length $\geq 2$. Again using lemmas 23 and 24, $G$ is reconstructible from $G_{v}$ where $v$ is on $P$, unless there is an E-vertex $v$ with 3 incident thin L-multipaths.

If $G$ has 2 fat multipaths, using lemma 23 and subtracting the L-multipaths of $G_{v}$, the multiset $\Sigma$ of L-multipaths incident to $v$ is known. If these all have size $0 G$ is reconstructible from $G_{v}$. If $\Sigma$ contains an L-multipath of length $\geq 3$ $G$ is reconstructible from $G_{w}$ where $w$ is on such a path and at a distance $\geq 2$ from $v$. Otherwise, $G$ is reconstructible from $G_{v}$.

Suppose there is a fat multipath $M$ and 5 thin multipaths, at least one of which has length $\geq 2$. The argument is as in this case of the proof of lemma 22 , except that $w$ is arbitrary and lemmas 23 and 24 are used.

Thus, it may be assumed that all thin L-multipath are edges.

If there is an E-vertex $v$ such that $G_{v}$ is a triangle with some limbs attached, using lemma 23, $G$ may be reconstructed from $G_{v}$.

If the L-multipaths which are edges form a square let $v$ be an interior vertex of a path on an L-multipath of multiplicity 2 ; then $G$ is reconstructible from $G_{v}$.

If the L-multipaths which are edges form a path of length 2 let $v$ be an Evertex such that $G_{v}$ is a triangle of fat L-multipaths with one E-vertex having a limb. Then $G$ is reconstructible from $G_{v}$. 
If the L-multipaths which are edges form a parallel pair let $v$ be an interior vertex of a path on an L-multipath of multiplicity 2 ; then $G$ is reconstructible from $G_{v}$.

Suppose there is a single L-multipaths which is an edge, with E-vertices $v_{1}, v_{2}$, and $v_{3}, v_{4}$ the remaining E-vertices. By an argument in the proof of lemma 18, adapted using lemmas 23 and 24, every fat multipath must contain an edge. By considering $G_{v}$ for $v \in\left\{v_{3}, v_{4}\right\}$, the multiset $\Sigma$ of L-multipaths incident to $v_{1}$ or $v_{2}$ may be determined. If an L-multipath of $\Sigma$ has a path of length $\geq 3$, let $M$ be one of least multiplicity among such, let $P$ be a path of $M$ of length $\geq 3$, and let $w$ be the vertex of $P$ such that in $G_{w}$ there is only one limb, and it is attached to $v_{3}$ or $v_{4} . M_{v}$ may be found in $G_{v}$, and $P$ may be determined, whence by lemma $24 G$ may be reconstructed.

If there is no such $M$ in $\Sigma$, let $M$ be the L-multipath with ends $v_{3}, v_{4}$. If $M$ has a path $P$ of length $\geq 3$ proceed similarly. Otherwise, all paths which are not edges have length 2 , and $G$ is reconstructible from $G_{v}$ where $v \in\left\{v_{3}, v_{4}\right\}$.

Lemma 26. If $G$ has no E-limbs, all L-multipaths are thin, and there are $\geq 2$ L-multipath of nonzero size, then $G$ is reconstructible.

Proof. By theorem 10.b the L-paths are known. Let $l_{0}$ be the minimum length of a size 0 L-path. Let $F$ denote the set of size 0 length $l_{0}$ paths.

If $F$ contains a triangle, i.e., there is an E-vertex $v$ such that in $G_{v}$ there is a cycle of length $3 l_{0}$ with 3 limbs attached, $G$ is reconstructible from $G_{v}$.

If $F$ is a square let $u$ be the degree 1 vertex of the I-limb of an L-path of size $1 ; G$ is reconstructible from $G_{u}$.

Suppose $F$ is a path of length 2. Number the E-vertices so that along the path they are 102, and 3 is the remaining one. Let $P$ be the $12 \mathrm{~L}$-path; $P$ is determined from any $G_{u}$ where $u$ is a degree 1 vertex of a limb not on $P$. If $P$ has size $\geq 2$ then $G$ is reconstructible using lemma 4 . Let $\Sigma$ be the L-paths incident to vertex 3 , with 3 marked. If $P$ has size $1 \Sigma$ is known from $G_{u}$ where $u$ is the degree 1 vertex of the limb of $P$; otherwise it may be determined by a standard argument from the $G_{u}$ for $u$ a degree 1 vertex. As noted in earlier arguments, it may be assumed that the 03 L-path has size 0 , and only one L-path in $\Sigma$ has nonzero size, say the 01 L-path. $G$ may be reconstructed from $G_{u}$ where $u$ is the degree 1 vertex of the I-limb of the 01 L-path.

Suppose $F$ is a parallel pair. If there is a path of length $\geq 3$ in $F^{\mathrm{cmpl}}$, the complement of $F$, let $w$ be a vertex on such a path $P$ such that $G_{w}$ has as few limbs as possible. In $G_{w}, 3$ of the E-vertices of $G$ may be found from degree considerations, and the 4 th using the fact that the two length $l_{0}$ L-paths are 
parallel; $G$ may now be reconstructed using lemma 24 .

Suppose all L-paths in $F^{\mathrm{cmpl}}$ have length 2 . If $l_{0} \geq 2$ there cannot be an L-path not in $F$ of size 0 , whence $G$ is reconstructible from $G_{u}$ for $u$ the degree 1 vertex of the limb on an L-path of size 1 . If $l_{0}=1$ there must be an L-path not in $F$ of size 0 ; let $w$ be its midpoint. $G$ is reconstructible from $G_{w}$, since on at least 1 path of length 3 the E-vertex may be located, and the other may then be by finding the parallel edge.

Suppose $F$ is a single L-path; let $\{0,1\}$ be the its incident E-vertices, and $\{2,3\}$ the E-vertices incident to the path $P$ parallel to the edge. If $P$ has size $\geq 2$ then $G$ is reconstructible using lemma 4 .

Suppose $P$ has size 1. If $P$ is symmetric then $G$ is reconstructible from $G_{u}$ where $u$ is the degree 1 vertex of the limb of $P$. Suppose $P$ is not symmetric. For $i \in\{2,3\}$ let $Q_{i}$ be the path between 0 and 1 containing $i$, with attached limbs, and let $d_{i j}$ be the distance from $i$ to $j, j \in\{0,1\}$. If $\left\{d_{20}, d_{21}\right\} \neq\left\{d_{30}, d_{31}\right\}$, let $u$ be the degree 1 vertex of the limb on $P$ and let $t$ be any other degree 1 vertex; the location of the limb on $P$ may be determined from $G_{t}$, and $G$ then reconstructed from $G_{u}$. Let $s_{i}$ be the size of $Q_{i}$, and assume $s_{2} \leq s_{3}$; if $0<s_{2}<s_{3}$ the argument is similar. The remaining cases are as follows, noting that $s_{2} \leq 2$ may be assumed.

01 . The limb on $Q_{3}$ is between $j$ and 3 for exactly one $j \in\{0,1\}$, and the distance from its root to $j$ is determined from $G_{u}$.

$0, \geq 2$. $G$ may be reconstructed using lemma 4 for $Q_{3}$.

11. Considering $Q_{i}$ as oriented, unless $Q_{2}=Q_{3}$ or $Q_{2}=Q_{3}^{r}$, the location of the limb on $P$ may be determined from the $G_{t}$. In the remaining cases the two possibilities are isomorphic.

22. Using lemma 4 it may be assumed that both $Q_{i}$ are symmetric. Unless $Q_{2}=Q_{3}$ the location of the limb on $P$ may be determined from the $G_{t}$, and in the remaining case the two possibilities are isomorphic.

Suppose $P$ has size 0 , and let 0,1 etc. be as above. if $P$ has length 2, which can occur only if $l_{0}=1$, then $Q_{2} \cdot Q_{3}$ are known from $G_{w}$ where $w$ is the midpoint of $P$. Unless $s_{2}=1$ and $s_{3} \leq 2 G$ is reconstructible by lemma 10 .

In case 11 of $s_{2}, s_{3}$, unless $Q_{2}=Q_{3}$ or $Q_{2}=Q_{3}^{r}, G$ is reconstructible from either $G_{t}$ using $G_{w}$, and in the remaining cases the two possibilities are isomorphic.

In case 12, unless $Q_{3}$ is symmetric and $Q_{2}$ is not $Q_{3 t}$ where $t$ is a degree 1 vertex of a limb on $Q_{3}, G$ is reconstructible from $G_{t}$ using $G_{w}$, where $t$ is the degree 1 vertex of the limb on $Q_{2}$. Unless $\left\{d_{20}, d_{21}\right\}$ equals $\left\{d_{30}, d_{31}\right\}, G$ may be reconstructed from $G_{t}$ for $t$ the degree 1 vertex of a limb of $Q_{3}$. If $d_{20}=d_{30}$ $G$ is reconstructible from $G_{t}$ where in $G_{t} Q_{2}=Q_{3}$ and if if $d_{20}=d_{31} G$ is 
reconstructible from $G_{t}$ where in $G_{t} Q_{2}=Q_{3}^{r}$.

Suppose $P$ has length $\geq 3$. If $2 \leq s_{2}<s_{3}, Q_{2}$ is known from $G_{w}$ where $w$ is a vertex on $P$ such that in $G_{w} Q_{2}$ has size $2 ; G$ may be reconstructed using lemma 4. If $2 \leq s_{2}=s_{3}, Q_{2}, Q_{3}$ are known from the $G_{w}$ where $w$ is a vertex on $P$ such that in $G_{w}$ either $Q_{2}$ or $Q_{3}$ has size $2 ; G$ is reconstructible by lemma 10. If $s_{2}=1$ and $s_{3} \geq 3, Q_{3}$ is known from $G_{t}$ where $t$ is the degree 1 vertex of the limb on $Q_{2} ; G$ may be reconstructed using lemma 10.

In case 11 of $s_{2}, s_{3}$, unless $\left\{d_{20}, d_{21}\right\}$ equals $\left\{d_{30}, d_{31}\right\}, G$ may be reconstructed from the $G_{t}$. This is also true unless $d_{20}=d_{21}$, and in that case $G$ is reconstructible from $G_{w}$ where $w$ is a vertex along $P$.

In case 12 , again $\left\{d_{20}, d_{21}\right\}=\left\{d_{30}, d_{31}\right\}$ may be assumed. Letting $w$ be the vertex on $P$ such that in $G_{w} Q_{3}$ has 3 limbs, and $t$ the degree 1 vertex of the limb on $Q_{2}, G_{w}$ lies over $G_{t}$ in only one way, unless $Q_{3}$ is symmetric and $v_{3}$ is the midpoint. In that case, $\left\{d_{20}, d_{21}\right\}$ is known from $G_{t}$ where $t$ is a degree 1 vertex of a limb on $Q_{3}$. $G$ may then be reconstructed from $G_{w}$ where $w$ is a vertex along $P$ such that in $G_{w} Q_{3}$ has 2 limbs.

Lemma 27. If $G$ has no E-limbs, all L-multipaths are thin, and there is only $1 \mathrm{~L}$-multipath of nonzero size, then $G$ is reconstructible.

Proof. Let $P$ be the path with limbs. Suppose there is a limb of size $\geq 2$. If there is only one limb $G$ is reconstructible. Otherwise let $R$ be $P$ with the roots of limbs marked; $R$ is determined from $G_{u}$ where $u$ is the degree 1 vertex of a limb of size $\geq 2$. Let $t$ be the degree 1 vertex of a limb of size 1 . Either $R$ fits over $P_{t}$ in only one way, or $R$ is symmetric; in either case $G$ is reconstructible from $G_{t}$.

Suppose $P$ has length $\geq 4$. Let $w$ be on $P$, such that in $G_{w}$ there are 2 limbs, one of size 1 , or one of size 2 if there is no $w$ with one of size 1 . $G$ is reconstructible from $G_{w}$. If $P$ has length 3 and there are 2 limbs, $G$ is reconstructible from $G_{u}$ where $u$ is a degree 1 vertex of a 1-limb.

If a size $0 \mathrm{~L}$ - path has length $\geq 6 G$ is reconstructible from $G_{w}$ where $w$ is at distance $\geq 3$ from both ends. There remain only a finite number of graphs; a computer program was written to verify that these all have distinct decks.

Theorem 28. If $G$ is an SBT graph with $B_{m}=K_{4}$ then $G$ is reconstructible.

Proof. This follows by lemmas 18 to 27 . 



Figure 1: S-minimal graphs for $n_{p}=3$

$$
\text { 9. } n_{p}=2
$$

Theorem 29. If $n_{p}=2$ then $G$ is reconstructible.

Proof. When $n_{p}=2$ there are 4 S-minimal graphs; see Figure 2 of [4]. From left to right the multipath graphs are an edge, a triangle, a square, and $K_{4}$. In the first case, $G$ is reconstructible by theorem 8 . In the second and third cases 
$G$ is reconstructible by theorem 17 . In the fourth case $G$ is reconstructible by theorem 28.

\section{Remarks on $n_{p}=3$}

Figure 1 shows the 17 S-minimal graphs for $n_{p}=3$, each given as its multipath graph, with multipath multiplicities indicated. Numbering from 1, left to right, top to bottom, SBT graphs with $B_{m}$ equaling graph 1 are reconstructible by theorem 8, those with $B_{m}$ equaling graph 2-7 are reconstructible by theorem 17, and those with $B_{m}$ equaling graph 14 are reconstructible by theorem 28 .

As noted earlier, any inseparable graph of size $\geq 2$ can occur as $B_{m}$. The simplest $B_{m}$ yet to be considered is that of graphs 10-11 of figure 1. It is clear, though, that even though the graph reconstruction conjecture seems likely to be true, the case-by-case methods of this paper are inadequate to make more substantial progress.

\section{References}

[1] J. A. Bondy, On Ulam's conjecture for separable graphs, Pacific J. Math. 31 (1969), 281-288. http://dx.doi.org/10.2140\%2Fpjm.1969.31.281

[2] M. Dowd, Strong Reconstructibility of the Block-Cutpoint Tree, Int. J. Pure Appl. Math. , 87, No. 4 (2013), 513-528 http://dx.doi.org/10.12732/ijpam.v87i4.2

[3] M. Dowd, Two Cases of Reconstruction of Separable Graphs, Int. J. Pure Appl. Math. , 90, No. 1 (2014), 25-34. http://dx.doi.org/10.12732/ijpam.v90i1.4

[4] M. Dowd, Some Results on Reconstructibility of Colored Graphs, Int. J. Pure Appl. Math. 95, no. 2 (2014), 309-321. http://dx.doi.org/10.12732/ijpam.v95i2.14

[5] B. D. McKay and A. Piperno, Practical Graph Isomorphism, II, J. Symbolic Computation 60 (2013), 94-112. http://dx.doi.org/10.1016/j.jsc.2013.09.003. 
